\title{
Current clinical application of traditional chinese medicine for the treatment of hypertension
}

\author{
Huai Wang ${ }^{1,2}$, Li-ya Bao $^{1,2}$, Xue-ling Zhou ${ }^{1,2}$, You-qin Chen ${ }^{4}$, Chang-geng Fu , Jian-feng Chu $^{1,2,3 *}$ and Jun Peng ${ }^{1,2,3 *}$ \\ ${ }^{1}$ Academy of Integrative Medicine, Fujian University of Traditional Chinese Medicine, Fuzhou, Fujian 350122, China \\ ${ }^{2}$ Fujian Key Laboratory of Integrative Medicine on Geriatrics, Fuzhou, Fujian 350122, China \\ ${ }^{3}$ Chen Keji Academic Thought Heritage Studio, Fujian University of Traditional Chinese Medicine, Fuzhou, Fujian 350122, China \\ ${ }^{4}$ Case Western Reserve University School of Medicine, Rainbow Babies and Children's Hospital, Cleveland, OH 44106, USA \\ ${ }^{5}$ Cardiovascular Diseases Center, Xiyuan Hospital of China Academy of Chinese Medical Sciences, Beijing 100091, China
}

\begin{abstract}
Background: Hypertension is the most common type of chronic non-communicable diseases, as well as being the single most important risk factor for cardiovascular disease. The use of TCM has long been used in the prevention and treatment of hypertension and is associated with effective outcomes.

Objective: Determine the current status in the clinical application of Traditional Chinese medicine(TCM) for the treatment of hypertension within the past 5 years.

Search strategy: Seven electronic databases (PubMed, EMbase, Cochrane library, QVID, China National Knowledge Infrastructure (CNKI), Chongqing VIP (CQVIP), Wanfang Data) were systematically searched from 2012-2016 using MeSH terms such as "hypertension, high blood pressure "and "Traditional Chinese medicine, Chinese medicinal herb, Chinese herbal medicine."

Inclusion criteria: Clinical randomized controlled trials, case-control studies, and case studies.

Data extraction and analysis: All statistics were retrieved based on the type of clinical study, type of prescription and type of treatment. Subsequently, the medicine meridian, efficacy of prescription and type of syndromes were summarized.

Results: Tianma Gouteng decoction and Zhengan Xifeng decoction were used 7 times, reinforcing deficiency was used 23 times, Calming the Liver was used 22 times and Removing Blood Stasis was used 14 times. Uncaria was used 24 times, Achyranthes and Tuckahoe were used 20 times, there are 50 kinds of drugs in the liver meridian. The main common clinical symptoms of hypertension were Hyperactivity of liver Yang and Yin deficiency and Yang Hyperactivity.

Conclusion: Recent clinical application of TCM for the treatment of hypertension most frequently involve the prescriptions Tianma Gouteng decoction and Zhengan Xifeng decoction, and the components Uncaria, Achyranthes and Tuckahoe. In TCM, occurrence of hypertension is mainly attributed to Hyperactivity of liver Yang, as well as Yin deficiency and Yang Hyperactivity, and treatment of hypertension most commonly involve reinforcing deficiency, Calming the Liver and Removing Blood Stasis.
\end{abstract}

\section{Introduction}

Current treatment of hypertension is significantly dominated by Western medicine. In comparison, TCM only plays an auxiliary function in the prevention and control of hypertension. However, TCM often demonstrates greater effectiveness during prehypertension, by inhibiting the development of high blood pressure, and other clinical symptoms such as dizziness, insomnia, and chest tightness, as well as providing improved treatment of refractory hypertension. Although TCM is commonly used for the clinical prevention and treatment of hypertension, there are often significant variations in the treatment effects in relation to the specific disease, pathogenesis, and type of medication. Thus, there is a lack of normative evaluation of various TCM prescriptions in relation to their curative treatment effects. This article examines the various controlled clinical trials of TCM within the past 5 years and analyzes the current TCM prescriptions in the prevention and treatment of hypertension.

\section{Methods}

We analyzed recent literatures involving TCM and clinical application of hypertension treatment using literature retrieval. Seven electronic databases (PubMed, EMbase, Cochrane library, QVID, China National Knowledge Infrastructure (CNKI), Chongqing VIP (CQVIP), Wanfang Data) were systematically searched from 2012-2016 using MeSH terms such as "hypertension, high blood pressure" and "Traditional Chinese medicine, Chinese medicinal herb, Chinese herbal medicine." and retrieved studies which used TCM in the treatment of hypertension, with the inclusion criteria for clinical randomized controlled trials, case-control studies, and case studies, and

*Correspondence to: Chu Jianfeng, Academy of Integrative Medicine, Fujian University of Traditional Chinese Medicine, 1 Qiuyang Road, Minhou Shangjie, Fuzhou, Fujian 350122, China, E-mail: jianfengchu@126.com

Peng Jun, Academy of Integrative Medicine, Fujian University of Traditional Chinese Medicine, 1 Qiuyang Road, Minhou Shangjie, Fuzhou, Fujian 350122, China, E-mail: pjunlab@hotmail.com

Key words: hypertension, traditional chinese medicine, clinical application

Received: December 05, 2018; Accepted: December 17, 2018; Published: December 19, 2018 
the exclusion criteria for non-clinical research involving in vitro and animal experiments. All statistics were retrieved based on the type of clinical study, type of prescription and type of treatment. Subsequently, the medicine meridian, efficacy of prescription and type of syndromes were summarized. In total, a total of 19 clinical TCM prescriptions were selected for the treatment of hypertension. Finally, we analyzed the composition, efficacy and indications of the different prescriptions. Based on these collected data, a database was setup and subsequent statistical analyses were performed.

\section{Results}

\section{Article retrieval and categorization}

A total of 32 articles were retrieved which met the criteria, including 29 clinical randomized controlled trials, 1 case-control study, and 2 case studies. In addition, 17 articles investigated well known TCM prescriptions (Tianma Gouteng decoction, Zhengan Xifeng decoction, Banxia Baizhu Tianma decoction, Xuefu Zhuyu decoction), whereas 15 articles investigated self-made prescriptions (Table 1) [1-32].

\section{Characteristics of TCM treatment prescriptions}

The frequency of use of compound TCMs in treatment of hypertension included: Tianma gouteng decoction-7 times, Zhengan Xifeng decoction-7 times, Banxia baizhu tianma decoction-2 times, and other formulae-1 time (Table 1). The most common treatment prescription characteristics included: reinforce deficiency-23 times, Calm the Liver-22 times, Remove Blood Stasis-14 times, Clear heat-12 times, expel dampness- 6 times, regulate flow of qi- 4 times, tranquilize endogenous wind-3 times, and Tranquilize-1 times (Table 2).

\section{Characteristics of hypertension}

The common clinical syndromes of hypertension included: Occurrences of Hyperactivity of liver Yang-10 times, Occurrences of Yin deficiency and Yang Hyperactivity-9 times, Occurrences of Blood stasis-7 times, Occurrences of Phlegm-damp resistance-6 times, Occurrences of Zangfu Qi-Xue and Yin-Yang deficiency- 5 times, Occurrences of Overabundant liver-fire-3 times, and Occurrences of Stagnated heat of liver meridian-2 times (Table 2).

\section{Characteristics of TCM components}

There was a total of 74 different types of TCM components used for the treatment of hypertension. Among these, the top three most commonly used TCM components were: Uncaria-24 times, Achyranthes-20, and Tuckahoe-20 times (Table 3).

The most common organ targets of TCM meridian included: 50 liver, 25 heart, 32 spleen, 30 lung, 18 kidney, 3 pericardial, 9 bile, 0 small intestine, 25 stomach, 9 large intestine, 5 bladder, and 1 triple energizer meridian (Table 4). When TCM usage was expanded to used more than 10 times, the most common organ targets of TCM meridian included: 9 liver, 10 heart, 5 kidney, 4 spleen, 4 lung, 2 pericardial, 3 bile, 0 small intestine, 3 stomach, 1 large intestine, 1 bladder, and 1 triple energizer meridian (Table 5). Subsequently, when TCM usage was further expanded to used more than 20 times, the most common organ targets of TCM meridian included: 2 liver, 2 heart, 2 kidney, 1 spleen, 1 lung, 0 pericardial, 0 bile, 0 small intestine, 0 stomach, 0 large intestine, 0 bladder, and 0 triple energizer meridian (Table 6).

Table 1. Prescription, Usage Counter, Study Types

\begin{tabular}{|c|c|c|c|c|c|}
\hline Prescription & Usage Counter & Study Types & Prescription & Usage Counter & Study Types \\
\hline Tianma Gouteng decoction [1-7] & 7 & $\begin{array}{c}7 \text { Clinical Randomized Controlled } \\
\text { Trial }\end{array}$ & Compound TCM [28] & 1 & $\begin{array}{l}\text { Clinical Randomized } \\
\text { Controlled Trial }\end{array}$ \\
\hline Zhengan Xifeng decoction [8-14] & 7 & $\begin{array}{c}5 \text { Clinical Randomized Controlled } \\
\text { Trial, } 1 \text { Case Study,1 Case-control } \\
\text { Studies }\end{array}$ & Antihypertensive Tea [30] & 1 & $\begin{array}{l}\text { Clinical Randomized } \\
\text { Controlled Trial }\end{array}$ \\
\hline $\begin{array}{l}\text { Banxia Baizhu Tianma decoction } \\
\qquad[16,17]\end{array}$ & 2 & $\begin{array}{l}2 \text { Clinical Randomized Controlled } \\
\text { Trial }\end{array}$ & Pingshen Tongluo Jiangya granula [22] & 1 & $\begin{array}{l}\text { Clinical Randomized } \\
\text { Controlled Trial }\end{array}$ \\
\hline Xuefu Zhuyu decoction [18] & 1 & $\begin{array}{c}\text { Clinical Randomized Controlled } \\
\text { Trial }\end{array}$ & Xinnao Xikang [24] & 1 & $\begin{array}{l}\text { Clinical Randomized } \\
\text { Controlled Trial }\end{array}$ \\
\hline Qingxuan Jiangya decoction [19] & 1 & $\begin{array}{c}\text { Clinical Randomized Controlled } \\
\text { Trial }\end{array}$ & Zhengan Xifeng Huatan Quyu TCM [26] & 1 & $\begin{array}{l}\text { Clinical Randomized } \\
\text { Controlled Trial }\end{array}$ \\
\hline Sancao Jiangya decoction [20] & 1 & Case Study & Compound TCM [27] & 1 & $\begin{array}{l}\text { Clinical Randomized } \\
\text { Controlled Trial }\end{array}$ \\
\hline Bushen Heluo fang [21] & 1 & $\begin{array}{c}\text { Clinical Randomized Controlled } \\
\text { Trial }\end{array}$ & Naoqingtong Granule [29] & 1 & $\begin{array}{l}\text { Clinical Randomized } \\
\text { Controlled Trial }\end{array}$ \\
\hline Huayu Xiezhuo decoction [23] & 1 & $\begin{array}{c}\text { Clinical Randomized Controlled } \\
\text { Trial }\end{array}$ & Pingganjiangya Granule [31] & 1 & $\begin{array}{l}\text { Clinical Randomized } \\
\text { Controlled Trial }\end{array}$ \\
\hline TCM Tea[25] & 1 & $\begin{array}{c}\text { Clinical Randomized Controlled } \\
\text { Trial }\end{array}$ & Sangji Mixture[32] & 1 & $\begin{array}{l}\text { Clinical Randomized } \\
\text { Controlled Trial }\end{array}$ \\
\hline Self-made TCM[15] & 1 & $\begin{array}{c}\text { Clinical Randomized Controlled } \\
\text { Trial }\end{array}$ & & & \\
\hline
\end{tabular}

Table 2. Efficacy Prescription, Usage Counter, Syndromes

\begin{tabular}{|c|c|c|c|}
\hline Efficacy Prescription & Usage Counter & Syndromes \\
\hline Reinforcing deficiency & 23 & Hyperactivity of liver Yang \\
\hline Calming the Liver & 22 & Yin deficiency and Yang Hyperactivity \\
\hline Removing Blood Stasis & 14 & Blood stasis \\
\hline Clearing heat & 12 & Phlegm-damp resistance \\
\hline Expelling dampness & 6 & Zangfu Qi-Xue and Yin-Yang deficiency & \\
\hline Regulating flow of qi & 4 & Overabundant liver-fire \\
\hline Tranquilizing endogenous wind & 3 & Stagnated heat of liver meridian \\
\hline Tranquilization & 1 & \\
\hline
\end{tabular}


Table 3. TCM Name, Usage Counter

\begin{tabular}{|c|c|c|c|c|c|c|c|}
\hline TCM Name & Usage Counter & TCM Name & Usage Counter & TCM Name & Usage Counter & TCM Name & Usage Counter \\
\hline Uncaria & 24 & Tortoise plastron & 7 & Fructus Aurantii & 2 & Rhizoma Dioscoreae & 1 \\
\hline Achyranthes & 20 & Hematite & 7 & $\begin{array}{l}\text { Fructus Ligustri } \\
\text { Lucidi }\end{array}$ & 2 & Scorpion & 1 \\
\hline Tuckahoe & 20 & Pinellia & 7 & Fructus Arctii & 2 & Lignum Sappan & 1 \\
\hline Scutellaria baicalensis & 18 & Fossil fragments & 6 & $\begin{array}{l}\text { Flos Chrysanthemi } \\
\text { Indici }\end{array}$ & 2 & Vervain & 1 \\
\hline Danshen Root & 15 & Oyster & 6 & ginger & 2 & Medulla Tetrapanacis & 1 \\
\hline Capejasmine & 13 & Crataegus pinnatifida & 4 & Fructus Jujubae & 2 & Pine needle & 1 \\
\hline Gastrodia elata & 12 & Chrysanthemum & 4 & Broadleaf holly leaf & 1 & Citron & 1 \\
\hline Parasitic loranthus & 12 & Radix Paeoniae Rubra & 4 & Rhizoma Coptidis & 1 & Greenbrier & 1 \\
\hline Rehmanniae & 11 & Alisma & 3 & Earthworm & 1 & $\begin{array}{l}\text { Officinal Magnolia } \\
\text { Bark }\end{array}$ & 1 \\
\hline Licorice & 11 & Rhubarb & 3 & Radish Seed & 1 & $\begin{array}{l}\text { Integripetal rhodiola } \\
\text { herb }\end{array}$ & 1 \\
\hline Chuanxiong & 11 & Tangerine peel & 3 & Rhizoma Polygonati & 1 & Citron fruit & 1 \\
\hline $\begin{array}{l}\text { Large-headed } \\
\text { atractylodes }\end{array}$ & 10 & Selfheal & 2 & Fushen & 1 & Bile Arisaema & 1 \\
\hline Radix Paeoniae Alba & 9 & Radix Puerariae & 2 & Chinese Angelica & 1 & Medicated leaven & 1 \\
\hline Motherwort & 9 & Pearl shell & 2 & $\begin{array}{l}\text { Fructus Aurantii } \\
\text { Immaturus }\end{array}$ & 1 & $\begin{array}{c}\text { Grassleaf Sweetflag } \\
\text { Rhizome }\end{array}$ & 1 \\
\hline Sickle senna seed & 9 & Herba Epimedii & 2 & Semen Persicae & 1 & $\begin{array}{l}\text { Barbary Wolfberry } \\
\text { Fruit }\end{array}$ & 1 \\
\hline Figwort & 9 & Leech & 2 & Radix Platycodi & 1 & Pipewort & 1 \\
\hline Concha Haliotidis & 8 & Radix Astragali & 2 & Chinese thorowax & 1 & Cassia & 1 \\
\hline $\begin{array}{l}\text { Caulis polygoni } \\
\text { multiflori }\end{array}$ & 8 & Folium Mori & 2 & Rough gentian & 1 & & \\
\hline Eucommia & 8 & Caulis Bambusae & 2 & Safflower & 1 & & \\
\hline
\end{tabular}

Table 4. Medicine Meridian

\begin{tabular}{|c|c|c|c|}
\hline Medicine Meridian & Usage Counter & Medicine Meridian \\
\hline Liver & 50 & Bile \\
\hline Heart & 25 & Small Intestine \\
\hline Spleen & 32 & Stomach \\
\hline Lung & 30 & Large Intestine \\
\hline Kidney & 18 & Bladder \\
\hline Pericardial & 3 & Triple Energizer & 25 \\
\hline
\end{tabular}

Table 5. Medicine Meridian, using more than 10 times (Contains 10)

\begin{tabular}{|c|c|c|c|}
\hline Medicine Meridian & Usage Counter & Medicine Meridian & Bile \\
\hline Liver & 9 & Small Intestine \\
\hline Heart & 10 & Stomach \\
\hline Kidney & 5 & Large Intestine \\
\hline Spleen & 4 & Bladder \\
\hline Lung & 4 & Triple Energizer \\
\hline Pericardial & 2 & 1 \\
\hline
\end{tabular}

Table 6. Medicine Meridian, using more than 20 times (Contains 20)

\begin{tabular}{|c|c|c|c|}
\hline Medicine Meridian & Usage Counter & Medicine Meridian \\
\hline Liver & 2 & Bile \\
\hline Heart & 2 & Small Intestine \\
\hline Kidney & 2 & Stomach \\
\hline Spleen & 1 & Large Intestine \\
\hline Lung & 1 & Bladder & 0 \\
\hline Pericardial & 0 & Triple Energizer & 0 \\
\hline
\end{tabular}

\section{Discussion}

Hypertension is the most common type of chronic noncommunicable diseases, as well as being the single most important risk factor for cardiovascular disease [33,34]. In TCM, hypertension is categorized as the Vertigo and Headache class of disease. The basic pathogenesis of hypertension in TCM is the result of Yin deficiency and
Yang Hyperactivity, as well as the general symptom of asthenia. The main cause of hypertension is emotional disturbance, improper diet, blood stasis, phlegm interference, as well as occurrence of Zangfu QiXue and Yin-Yang deficiency in the liver and kidneys. The use of TCM has long been used in the prevention and treatment of hypertension and is associated with effective outcomes. 
The antihypertensive effects of TCM meridian was previously summarized by Huang Xiaojin [35], in particular the efficacy of TCM in the treatment of hypertension. The TCM notion for treatment of hypertension generally involves the five organs Yin principle, while its etiology and pathogenesis is mainly based on phlegm, blood stasis and heat accumulation. The law of frequency in the use of TCM for treatment of primary hypertension was outlined by Cai Yongmin [36] and involves the 6 common applications of TCM including: Reinforce deficiency, Remove Blood Stasis, Clear heat, Calm the Liver with Tranquilize endogenous wind, diaphoresis relieving superficies and Expel dampness. Studies by Lan Hongbin [37] and Chen Jianxin [38] analyzed 864 and 310 different compounds, respectively, for the treatment of hypertension, which includes 231 and 84 different kinds of TCM, respectively, whereby the most frequently used characteristics were: Reinforce deficiency, Remove Blood Stasis, Calm the Liver and Expel dampness.

Our present study demonstrated that based on the choice of prescriptions for the treatment for hypertension, Tianma gouteng decoction and zhengan Xifeng decoction were the preferred options, whose characteristics mainly include: Reinforce deficiency, Calm the liver and Remove blood stasis. In addition, based on the choice of TCM components for the treatment for hypertension, Uncaria, Achyranthes and Scutellaria baicalensis, were the most widely used, and are mainly dominated by the TCM liver meridian. Similarly, the three most commonly used TCM meridians include the liver, heart and kidney meridian drugs. Furthermore, the main clinical syndromes of hypertension were: Hyperactivity of liver Yang, Yin deficiency and Yang Hyperactivity.

\section{Conclusion}

In conclusion, Hyperactivity of liver Yang and Yin deficiency, and Yang Hyperactivity are the two major syndromes of hypertension, while TCM treatments mainly focus on reinforcing deficiency, Calming the Liver and Removing Blood Stasis in the treatment of hypertension. The most commonly used formulas for clinical treatment of hypertension are Tianma Gouteng decoction and Zhengan Xifeng decoction, whereas Uncaria, Achyranthes and Tuckahoe are the most commonly used TCM components for clinical treatment of hypertension. TCM drugs belong mainly to the liver meridian, while TCM drugs for the treatment of hypertension mostly belong to the liver, heart and kidney meridians.

\section{Source(s) of financial support of the study}

1. Fujian Provincial Health and Family Planning Commission, 2018CX-42.

2. Fujian Provincial Department of Science and Technology, 2018J01884.

3. National Natural Science Foundation of China, 81774135.

\section{References}

1. Li WL, Fan YL, Cai MX (2015) The intervention of Tianma Gouteng Decoction to hypertension myocardial fibrosis and its effect on S100A4. GuangZhou Yi Ke Da Xue Хие Вао 43: 45-49.

2. Zhu WD (2013) Tianma Gouteng Decoction intervened hypertension left ventricular remodeling of congestive heart failure and cardiac function in 76 cases. ZhongGuo Zhong Yi Yao Xian Dai Yuan Cheng Jiao Yu 11: 20-22.

3. Liu YY, Liu Y, Ke YF (2015) Effect of Tianma Gouteng Decoction on RSS system in patients with hypertensive disorder complicating pregnancy. ZhongGuo Zhong Yi Ji Zheng 24: 145-146.

4. Yin JY (2012) Tianma Gouteng Decoction in treating 60 cases of hypertension phase I and II. XianDai Zhong Xi Yi Jie He Za Zhi 21: 407-408.
5. Pu JC (2012) Hyperactivity hypertension treated by Tianma Gouteng Decoction. ZhongHua Zhong Yi Yao Xue Kan 30: 215-216.

6. Sha KC (2015) Observe the clinical efficacy of Tianma Gouteng Decoction on treatment of hyperactivity of liver yang hypertension. ZhongGuo Yi Yao Zhi Nan 13: 29-30.

7. Fu YG (2016) Effect of Tianma Gouteng Decoction in treating senile hypertension and its influence on serum CRP, TNF- $\alpha$ and renal function. SiChuan Zhong Yi 34: 78-80.

8. Yang JX (2016) Clinical research on treating hypertension with Zhengan Xifeng Decoction. ZhongYi Lin Chuang Yan Jiu 8: 15-16.

9. Qiu DW (2015) Zhengan Xifeng Decoction for treating primary hypertension. Zhong Yi Lin Chuang Yan Jiu 7: 73-74.

10. Li XT (2015) Zhengan Xifeng Decoction in treating 100 cases of hypertension. GuangMing Zhong Yi 30 :1457-1458.

11. Li ZR (2015) Zhengan Xifeng Decoction in treating hypertension:analysis of 60 cases QingHai Yi Yao Za Zhi 45: 67-68.

12. Gu P, Zhang MX (2016) Zhengan Xifeng Decoction in treating plasma metabonomics of yin deficiency and yang hyperactivity type of hypertension. LiaoNing Zhong $\mathrm{Yi} \mathrm{Za}$ Zhi 43: 1353-1355.

13. Li GY, Zheng XQ (2012) Clinical observation of treating 86 cases of essential hypertension with Zhengan Xifeng Decoction. ZhongYi Lin Chuang Yan Jiu 4: 65-66.

14. Bai JF, Huang X, Yan ZM (2016) Zhengan Xifeng Decoction in treating 45 cases of moderate primary hypertension. HuanQiu Zhong Yi Yao 9: 1105-1107.

15. Hu XW (2014) Clinical analysis of Traditional Chinese Medicine in treating obstinate hypertension. ZhongGuo Yi Yao Zhi Nan 12: 179-180.

16. Yuan RH, Wu QA (2016) Banxia Baizhu Tianma Decoction in treating 50 cases of hypertension. HeNan Zhong Yi 36: 1919-1921.

17. Fan MM, Wang YP (2015) Clinical observation on treating 62 cases of senile hypertension with the Banxia Baizhu Tianma Decoction plus the Taohong Siwu Decoction. ZhongYi Lin Chuang Yan Jiu 7: 115-116.

18. Sun XH, Chen XB (2016) Xuefu Zhuyu Decoction in the treatment of hypertension with left ventricular hypertrophy hypersensitive c-reactive protein and the effect of angiotensin II. ShanXi Zhong Yi 37: 1455-1457.

19. Yi L, Li J (2016) Effect and mechanism of Qingxuan Jiangya Decoction on hypertension patients with liver yang hyperactivity. LiaoNing Zhong Yi Yao Da Xue Xue Bao 18: 151153. [Crossref]

20. Fu B, Wang K (2014) Treating 61 cases of arthritis hypertensive vertigo with the Sancao Jiangya Decoction. ZhongYi Lin Chuang Yan Jiu 6: 78-79.

21. Liu YL (2012) Bushen Heluo Fang on clinical analysis of ventricular remodeling in essential hypertension. HeBei Yi Yao 34: 943-944.

22. Cui YH, Mou LM, Liu RR (2015) Observation of Pingshen Tongluo Jiangya Granule on the treatment of essential hypertension with early renal damage. HeBei Zhong Yi 37: 353-356.

23. Zeng LQ (2012) Clinical observation on treating 32 cases of hypertensive nephropathy in Traditional Chinese Medicine Huayu Lishui. ZhongYi Lin Chuang Yan Jiu 4: 18-19.

24. Ai J, Bian JP, Lu SS, Wang ZJ (2016) Xinnao Xikang in treatment of essential hypertension (Yin deficiency and Yang hyperactivity) clinical evaluation. XinJiang Zhong Yi Yao 34: 1-5.

25. Zheng BH, Lin S, Zheng MQ, Zheng Y, Zheng XP, et al. (2012) Clinical observation of Herbal Teas in the treatment of hypertension. ZhongGuo Lao Nian Bao Jian Yi Xue 10: 38-39.

26. Zheng WX (2016) Clinical analysis on treatment of hypertension by Zhengan Xifeng Huatan Quyu Traditional Chinese Medicine. HaiXia Yao Xue 28: 123-124.

27. Bi XB, Li J, Niu ML, Ma JY, Shang SY (2016) Observation on effect of compound Chinese Medicine in treatment of essential hypertension. KeJi Zhan Wang 26: 259-261.

28. Liu SX (2013) Observation on effect of Herbal Compound Formulae in treating essential hypertension. NeiMengGu Zhong Yi Yao 32: 23-65.

29. Zhou HZ, Li J (2015) Effects of Naoqingtong Granule on blood pressure, blood fat and hemorheology in patients with hypertension with liver heart phlegm stagnation and blood stasis syndrome. ZhongHua Zhong Yi Yao Za Zhi 30: 629-631.

30. Shang XY, Shi J, Hu YH (2014) Clinical observation on Antihypertensive Tea for Treatment of Prehypertensive Patients with Stagnated Heart of Liver Meridian Syndrome. ZhongXi Yi Jie He Xin Nao Xue Guan Bing Za Zhi 12: 385-387. 
31. Lin JM, Guo WX, Li YL (2012) Clinical research of Pinggan Jiangya Granule on treatment of hypertension liver-yang hyperactivity syndrome. ZhongHua Zhong Yi Yao Za Zhi 27: 1205-1208.

32. Zuo KK, Zhang MJ, Gu N (2014) Clinical research of Sangji Mixture on the immunological and inflammatory reaction in hypertension of overabundant liver-fire with phlegm syndrome. ZhongHua Zhong Yi Yao Za Zhi 29: 1649-1652.

33. Chen WW, Gao RL, Liu LS, Zhu ML, Wang W, et al. (2016) China cardiovascular disease summary report 2015. J Geriatr Cardiol 14: 1-10. [Crossref]

34. Moura NG, Cordovil I, Ferreira Ade S (2016) Traditional Chinese medicine wrist pulsetaking is associated with pulse waveform analysis and hemodynamics in hypertension. J Integr Med 14: 100-113. [Crossref]
35. Huang XJ, Liu WF, Sun YT, Xia LX, He LQ, et al. (2012) Discussion on rules of antihypertensive materia medica. ShangHai Zhong Yi Yao Za Zhi 46: 75-78.

36. Cai YM, Wang SF, Huang QF, Guo WJ (2014) Study on modern application frequency of Chinese Medicine in primary hypertension treatment. ShiJie Ke Xue Ji Shu-Zhong Yi Yao Xian Dai Hua 16: 603-606.

37. Lan HB, Yuan HP (2014) Analysis on Traditional Chinese Medicine treatment of hypertension medication rules. NeiMengGu Zhong Yi Yao 33: 75.

38. Chen JX, Xue CX, He J (2012) Investigation and analysis of Compound Chinese Medicine in treatment of senile hypertension medication rules. ZhongGuo Yi Yao Zhi Nan 10: 591-592.

Copyright: (C2018 Wang H. This is an open-access article distributed under the terms of the Creative Commons Attribution License, which permits unrestricted use, distribution, and reproduction in any medium, provided the original author and source are credited. 